\title{
The Role of Microglia in Cerebral Traumatic Injury and its Therapeutic Implications
}

\author{
Huber S. Padilla-Zambrano ${ }^{1}$ Harsh Deora2, ${ }^{-\infty}$ Mohamed Arnout ${ }^{3} \quad$ Romario Mendoza-Florez ${ }^{1}$ \\ Wiston Eduardo Cardenas-Chavez ${ }^{1}$ Monica Patricia Herrera-Martinez ${ }^{1}$ \\ Michael Gregorio Ortega-Sierra ${ }^{4}$ Amit Agrawal ${ }^{5}$ Luis Rafael Moscote-Salazar ${ }^{1}$
}

\author{
${ }^{1}$ Biomedical Research Center (CIB), Cartagena Neurotrauma \\ Research Group Research Line, Faculty of Medicine, University of \\ Cartagena, Cartagena, Colombia \\ 2Department of Neurosurgery, National Institute of Mental Health \\ and Neurosciences (NIMHANS), Bangalore, India \\ ${ }^{3}$ Zagazig University, Zagazig, Egypt \\ ${ }^{4}$ Rafael Núñez University Corporation, Cartagena de Indias, Colombia \\ ${ }^{5}$ Department of Neurosurgery, All India Institute of Medical \\ Sciences, Bhopal, Madhya Pradesh, India
}

\begin{abstract}
Address for correspondence Luis Rafael Moscote-Salazar, MD, Neurosurgeon-Critical Care, Biomedical Research Center (CIB), Director of the research line - Cartagena Neurotrauma Research Group, Faculty of Medicine - University of Cartagena, Cartagena de Indias, Bolívar, Colombia (e-mail: rafaelmoscote21@gmail.com).
\end{abstract}

Indian J Neurotrauma:2020;17:69-73

\begin{abstract}
Keywords

- microglia

- traumatic brain injury

- inflammation

- neurodegeneration

- diffuse axonal injury

Microglia have a variety of functions in the brain such as synaptic remodeling, damage repair of the central nervous system (CNS), and CNS' inflammatory response to peripheral infections. The response depends on the type of insult and infection and includes a range of variety of activation states, the duration of which will decide the outcome. In response to traumatic brain injury ( $\mathrm{TBI}$ ), early activation can lead to early restoration of function, while prolonged and continuous activation can cause neurodegeneration states. Current evidence, however, states that this may not be the case. In this article, we discuss this seldom understood topic of microglia response to TBI, and analyze their distribution, function and possible sites of manipulation. Animal studies have allowed genetic and pharmacological manipulations of microglia activation, in order to define their role. Microglia activation can be remote to the site of injury, and thus their manipulation may play a significant role in the response to any trauma.
\end{abstract}

\section{Introduction}

Microglia is a type of macrophage located in the central nervous system (CNS), derived from the mesoderm, ${ }^{1,2}$ which exercises glial functions and plays a role in immune defense. ${ }^{3}$ They represent approximately $15 \%$ of brain cells ${ }^{4}$ and are located mainly in the gray matter, hippocampus and basal ganglia. ${ }^{25,6}$ It is classified according to its status, resting or activated microglia, ${ }^{4,5}$ and participates in the mechanisms of injury and neuroprotection. ${ }^{5}$ The microglial response is important for the development, functioning ${ }^{1,2}$ and neuroprotection of the CNS secondary trauma, stroke, infections, and neurodegeneration. ${ }^{5}$ Also, they can self-renew, which confers the maintenance of this cell population during life. ${ }^{5,6}$ This article describes the generalities of microglia, its role in the CNS and traumatic brain injury (TBI).

\section{Microglia}

The microglia was first described in 1919 by Pio del Rio Hortega, ${ }^{7}$ who identified migrating phagocytic cells within the $\mathrm{CNS}^{2,6}$ and made a morphological and functional distinction between astrocytes, microglia, and oligodendrocytes. ${ }^{6,7}$ Kreutzberg, years later, described its activation process. ${ }^{8}$ Microglia are the macrophages and mediators of the CNS immune response to parenchymal infection and injury. ${ }^{7}$ They constitute approximately 10 to $15 \%$ of the brain cells, with predominance in the gray matter, ${ }^{2,5,6}$ hippocampus, basal ganglia, and olfactory cortex. ${ }^{5}$ Within its main functions are the immune defense, ${ }^{3,4,9}$ control of proliferation and neuronal differentiation, influences synaptic connections, remodeling of neuronal circuits, ${ }^{2,10}$ phagocytosis, ${ }^{4}$ and neuroprotection. ${ }^{1}$ It also has a capacity for self-renewal, thus contributing to
License terms India

C2020 Neurotrauma Society of
DOI https://doi.org/

10.1055/s-0040-1713078 ISSN 0973-0508. 
the maintenance of CNS homeostasis throughout life..$^{2,4,5}$ Two origins of the microglia have been considered: yolk sac ${ }^{5,6}$ and myeloid precursors installed in the $\mathrm{CNS}^{6}$ before the formation of the blood-brain barrier (BBB). ${ }^{10}$ These brain macrophages have been classified on the basis of their state: microglia at rest, with a branched morphology which allows constant inspection of the environment; and activated microglia, with amoeboid morphology that facilitates their participation in the mechanisms of injury and neuroprotection by exerting cytotoxic, proinflammatory functions and repair. ${ }^{5,10}$

Microglia show diverse phenotypic similarities with other cell types: tissue macrophages and circulating monocytes. Within these characteristics, the expression of several markers in immunohistochemical tests such as the receptor of the colony-stimulating factor, surface glycoproteins $F 4 / 80$, inhibitory immune receptor CD200R, calcium-binding protein lba-1, and others stand out, more specifically for microglia such as CD39 endonucleotidase; also, it has been reported that they may present a unique transcriptomic form which distinguishes it from other CNS cells, monocytes, and macrophages. ${ }^{2}$

For the initiation of microglial phagocytosis, it is necessary to activate several specific receptors located in the extracellular space and adjacent cell surfaces, which interact with pathogenic microorganisms and cell debris such as Toll-like receptors (TLRs). They are activated in cases of brain damage by stimulating the action of innate immunity: purinergic receptors (P2X7, P2Y12, P2X4 and adenosine receptors); peroxisome proliferator-activated receptors, which drive the concentration of activated microglia in the ischemic zones; and triggering receptors expressed in myeloid cells. ${ }^{4}$

\section{The Role of Microglia in the Central Nervous System}

As mentioned, microglia is an indispensable cellular group in homeostasis and protection against pathologies of the CNS., ${ }^{2,10,11}$ It participates in multiple processes such as immunity, aging, development, and regulation of the neuronal circuit,, ${ }^{2,10-12}$ and deregulation of these can lead to a disease's onset. ${ }^{11}$

During the neuronal differentiation and consolidation of neuronal circuits, any change in the microenvironment is detected by the microglia through its ramifications that allow constant monitoring of the environment, which leads to its activation that, depending on its regulation, may represent neuroprotection or, if its function is altered, lead to neurotoxicity. ${ }^{2}$ The microglia then shows various functional behaviors: CNS development, phagocytosis, and neuroprotection. ${ }^{6,10}$

\section{Microglia and the Neuronal Circuit}

Microglia interacts with multiple factors and the result depends on their variability. ${ }^{2}$ During the perinatal period, microglia's population increases considerably and the morphology of their branches changes progressively until the postpartum. This is associated with other changes in the
CNS, such as a decrease in apoptosis and the formation of synaptic circuits, contributing to neuronal development. ${ }^{6}$

During embryonic life, the activation of microglia can also be influenced by antigens such as lipopolysaccharides (LPS), interferon-gamma (IFN $\gamma$ ), or different drugs, releasing reactive oxygen species (ERO), tumor necrosis factor- $\alpha$ (TNF- $\alpha$ ), among other neurotoxic low-weight molecular factors and IL-6, which is reported by in vitro experiments to lead to the apoptosis of neuroblasts, decreasing neurogenesis in the embryo. ${ }^{8,13}$

In this same period, neurogenesis is accelerated for the development of the cerebral cortex, where the neural precursor of cells is subsequently phagocytosed to maintain homeostasis. ${ }^{8,10}$ In postnatal life, it continues to regulate neuronal circuits, phagocytizing senescent neurons by the expression of transforming growth factor $\beta$ (TGF- $\beta$ ), TNF- $\alpha$, endothelial growth factor, insulin-like growth factor 1 (IGF1), and TLR., ${ }^{2,8}$

Another important molecule for nerve development is CX3CL, a cytokine produced by neurons which interacts with microglia, and in its deficiency, there is reduced connectivity between different structures of the CNS which can impact behavior. $^{8}$

Microglia also modifies and eliminates synaptic structures when there are a lot of them, encapsulating the neuronal cells and displacing the synapses. This process is known as synaptic stripping, which is mediated by the electrical activity, since it induces the activation of the NMDA receptors ( $\mathrm{N}$-methyl-D-aspartate) in the neurons, allowing the influx of $\mathrm{Ca} 2+$ which activates the signals dependent on intracellular $\mathrm{Ca}^{2+}$ and ends in a de novo synthesis of neuroprotective and antiapoptotic molecules. Moreover, activated NMDA receptors induce the expression of the NFI-A factor (nuclear factor I-subtype A) coding gene, promoting neuronal survival. ${ }^{8,10} \mathrm{Also}$, it is mediated by the expression of complement factor $\mathrm{C} 1 \mathrm{q}$ and $\mathrm{C} 3$ by neurons, and the expression of the complement receptor CD11b / CD18 in microglia, ${ }^{11}$ eliminating selective axosomatic inhibitory synapses in adulthood and leaving the excitatory intact. This is because the microglia, through its receptors for the neurotransmitter GABA and glutamate, detects the diminished or altered activity of the inhibitory synapses as well as those which occurred in the period of CNS development with the over excitatory synapses. ${ }^{8}$

Microglia are located near blood vessels facilitating angiogenesis in neuronal development, ${ }^{2}$ A very important factor, as the PU.1 expressed in the microglia at rest and the activated state, is the regulator of the ontology of the lymphoid and myeloid hematopoietic cells line. ${ }^{11}$ It has been shown that a deficiency in this regulator affects the vascular structure and significantly decreases the population of mature $B$ cells and macrophages, because it is necessary for the expression of myeloid differentiation markers such as CSF (colonystimulating factor 1 ) which also contribute to cell survival, differentiation, and development of microglia. ${ }^{11,14}$ Another factor that allows the development of microglia is the regulatory factor of interferon-8 (IRF-8). ${ }^{2}$

The CSF1 factor has been widely studied for its importance in microglial differentiation. However, it is not a determinant 
agent due to the existence of IL-34 as a ligand that has a greater affinity to CSF1's receptor, suggesting variations of homeostasis in the macrophages cell line. This interleukin presents a pattern and moment of an expression that differs from the CSF1's, allowing a complementary receptor activation during the embryonic period and in adulthood.,

\section{Microglia as the Sentinel of the CNS}

Microglia has a role as a phagocyte in the CNS which helps to maintain an optimal and balanced environment. They can detect infiltrating immune cells, cells that have passed or are close to its programmed cell death, and structures such as synapses when those connections are not being useful or rapid enough and could hurt brain functioning. ${ }^{10}$ They can also induce their elimination through the release of cytokines such as TNF- $\alpha$, production of superoxide- 1 , and encapsulation, which represents a benefit even in neurodegenerative diseases..$^{8,10}$

Likewise, mutations in microglia receptors such as Trem-2 (activation receptor expressed in myeloid line), which are important in the mediation of phagocytosis, are associated with an increased risk of Alzheimer's disease because there is an increase in the extracellular deposition of $\beta$-amyloid plaques. $^{10}$

\section{Microglia and Neuroprotection}

The microglia activation has been considered a negative event due to the neurotoxicity that it represents itself; however, they present a great neuroprotective activity, reviewing the nervous parenchyma changes, suppressing the inflammation, and finally protecting the tissue from damage. Even though inflammation is a defense response, sometimes it could be exaggerated, generating more damage. ${ }^{10}$ For example, in meningitis, microglia initially releases IL-6 in response to infection, and then it produces an anti-inflammatory cytokine (IL-10) and TGF- $\beta$ in significant amounts as negative feedback, which contributes to nerve regeneration. . $^{8,10,12}$

Besides, it has been observed in several studies with mice that microglia provides support to neurons through the endothelial differentiation factor-1 (EDF-1), wherein a decrease in the proliferation of microglia leads to a worse prognosis in the face of an event of neuronal disease; 4 besides, with the help of this microglia-neuron interaction, a neurotropic activity that helps the neuron to recover from the damage is shared. ${ }^{13}$

\section{The Specific Role of Microglia in Cerebral Trauma}

When an external mechanical force hits the skull and exceeds its protective capacity, a TBI occurs. ${ }^{15}$ The initial trauma can produce direct damage by tearing, stretching, and neuronal and vascular shearing (with the consequent formation of hemorrhages and bruises). ${ }^{15,16}$ This is called a primary lesion. The primary lesion, in turn, unleashes the second production of biochemical mediators with the ability to induce shortand long-term cellular and metabolic changes within the nervous system. ${ }^{16,17}$ This reaction begins early from seconds to minutes after the trauma and can be sustained over days, months, and even years, ${ }^{17,18}$ which is called a secondary lesion. In this context, neuroinflammation, excitotoxicity, oxidative stress, disruption of the BBB, among others, are characteristic. ${ }^{15,16,19}$

Of all the mechanisms of a secondary lesion, neuroinflammation is the most important and can somehow regulate the sequelae after a $\mathrm{TBI}^{20}$ Microglia is the central regulator of neuroinflammation. ${ }^{21}$ The cells that constitute microglia represent the first line of defense against a lesion of the CNS, as the intermediaries of the innate immune system are located there. ${ }^{17,22}$ Once the trauma has occurred, the brain promotes a very complex immunological tissue reaction, evidenced in experimental animal models where various biomarkers of microglia activation, stress response and chemokine expression after brain trauma have been found. ${ }^{23,24}$ The activation of microglia is a process where these cells acquire different phenotypes, each with different functions, in response to specific signals. ${ }^{23}$ Three physiological states or phenotypes have been described in microglial cells. Normally, they exist in a state of rest, with branched form, known as phenotype I. Once neurological aggression occurs, the interstitial microenvironment is invaded by a series of biochemical signals derived from the surrounding cells, capable of inducing changes in the microglia, and undergoing a process of activation that can follow two routes: first, where the cell acquires the property to present antigens in the inflammation zones (phenotype II or M1), and second, where they turn into reactive cells, with phagocytic activity, and present in areas of trauma or infection. Recent studies have shown that cells can move from one specific phenotype to another. In the presence of CD40L, the microglia are established and maintained in the M1 phenotype; however, it can be transformed into the M2 phenotype through the CD45.

Several studies suggest that, like macrophages, microglia can rapidly move to the site of the initial lesion, as a large number of intracellular components of neurons and surrounding cells, known as damage-associated molecular patterns (DAMPs), are released in the primary lesion. These molecules include some excitatory neurotransmitters (mainly glutamate) that increase the flow of cations such as $\mathrm{Na}^{+}, \mathrm{Ca}^{2+}$, and $\mathrm{K+}$; ATP and substances to which microglia seem to be very sensitive to., ${ }^{7,22}$ The secretion by peripheral neutrophils that invade the site of the lesion can also trigger the activation. ${ }^{20}$ At that time, the activated microglia acquires a predominantly proinflammatory profile, with polarization toward M1 phenotype, with transcriptional activation of the nuclear factor-kB (NF-kB) and massive secretion of cytokines such as IL-1, IL-12, TNF- $\alpha$, and INF$\gamma$, and other neurotoxic substances such as reactive oxygen species, inducing oxidative stress.,23,25,26 Furthermore, as mentioned above, they acquire the function of antigen-presenting cells, expressing MHC-II, and promote cellular immunity. ${ }^{23}$ Some cells differentiate toward the M2 phenotype, which is characterized by the secretion of low levels of IL-4, IL-13, and IL-10, capable of inhibiting or mitigating the inflammatory process and favoring tissue repair and wound healing. It would be very beneficial to develop treatments 
that can enhance the healing properties of microglial activation, and at the same time, inhibit selectively the harmful effects of the same. ${ }^{7}$

Under normal conditions, the BBB prevents the entry of leukocytes into the CNS. However, after a trauma, the barrier gets altered, allowing the passage of cells and molecules into the CNS. ${ }^{27}$ In animal models, it has been discovered that there is a direct relationship between the severity of the trauma and the number of leukocytes that migrate to the CNS, and that monocytes and their derivatives constitute the largest population of these cells. ${ }^{28}$ These findings suggest that in addition to microglia, monocytes and macrophages may play a key role in the pathogenesis of neurological trauma and neuronal repair. ${ }^{27,28}$ Although these cell types are embryologically different, they are morphologically and functionally similar, so it is very difficult to isolate each type in the laboratory. They are known to share cell surface markers such as CD68, CD45, and MHC-II. This limitation to distinguish these two cell lines in a fast and reliable way has hindered the understanding of their interactions in neurological pathology. ${ }^{23,27}$

On the other hand, microglia seem to have a great influence on long-term neurological functioning. Neuroinflammation, where microglia are key components, is a common response to any type of neurological injury. This response in the acute and subacute phases is likely to be protective, however, there is much evidence in the literature that establishes a considerable association between the activation of prolonged or chronic microglia and cognitive neurological impairment. ${ }^{29,30}$ Also, it has been discovered that after cerebral trauma, there is an elevation of $\beta$-amyloid deposits in the CNS. These plaques, also found in Alzheimer's disease, can develop a few hours after the trauma. Also, there is an association with the increase of the enzymes necessary for the synthesis of amyloid- $\beta$ such as $\beta$-secretase (BACE1 protein) and proteins of the $\gamma$-secretase complex. Therefore, microglia may have a role in the accumulation and elimination of amyloid- $\beta .{ }^{17}$ However, a more in-depth research is needed to establish this relationship.

\section{Potential Therapeutic Targets Utilizing Microglia in TBI}

\section{Progranulin}

Progranulin is a neurotrophic and anti-inflammatory glycoprotein. It thus attenuates neuronal damage and microglia/macrophage activation in brain injury, but its mechanisms are still elusive. Intraventricular administration of recombinant progranulin in mice immediately before trauma reduced brain damage and neurological deficits, and restored normal levels of cytokine transcription, axonal injury, and astrogliosis 5 days after TBI in granulin knockout mice. ${ }^{31}$

\section{Minocycline}

This tetracycline has been known to have anti-inflammatory and neuroprotective properties via the inhibitory effects on the activities of key enzymes such as iNOS, MMPs, and PLA2. It has shown to be effective in acute treatment of TBI while not being so in chronic therapy in an animal model of TBI (closed head controlled cortical impact [CCI] in the neonate rat). ${ }^{32}$ However, in other TBI models, such as blast injury, minocycline appears to prevent the development of neurobehavioral abnormalities. ${ }^{33}$

\section{PPAR Agonist}

Fenofibrate, a known proliferator-activated receptor (PPAR) agonist, is one of the most promising compounds to offer neuroprotection. In adult male Sprague-Dawley rats, administration of fenofibrate during a clinically relevant therapeutic "time window of opportunity" at 1 hour after trauma mediated a significant posttraumatic neuroprotection. This was demonstrated by improved neurological scores in the fenofibrate group at 24 hours and 7 days after trauma, compared with vehicle-treated animals. ${ }^{34}$

\section{HDAC Inhibition}

Scriptaid is a class I/II HDACs, which was shown to facilitate and enhance recovery of motor functions after $\mathrm{CCI}$ and protected white matter up to 35 days after TBI, as shown by reductions in abnormally dephosphorylated neurofilament protein, increases in myelin basic protein, anatomic preservation of myelinated axons, and improved nerve conduction. ${ }^{35}$ It shifts microglia/macrophage polarization toward the protective M2 phenotype and mitigated inflammation.

\section{Conclusion}

The broader knowledge of the functions and interactions of microglia in traumatic neurological pathology could allow the development of new therapeutic measures that help to regulate the entire process of secondary injury in the shortand long-term in patients with neurological trauma. Besides, the development of biomarkers could optimize these treatments by predicting the ideal time for their use.

Funding

None.

Conflict of Interest

None declared.

\section{References}

1 Cullheim S, Thams S. The microglial networks of the brain and their role in neuronal network plasticity after lesion. Brain Res Brain Res Rev 2007;55(1):89-96

2 Ginhoux F, Prinz M. Origin of microglia : current concepts and past controversies. Cold Spring Harb Perspect Biol 2017;1-16

3 Casano AM, Peri F. Microglia: multitasking specialists of the brain. Dev Cell 2015;32(4):469-477

$4 \mathrm{Kim}$ J-Y, Kim N, Yenari MA. Mechanisms and potential therapeutic applications of microglial activation after brain injury. CNS Neurosci Ther 2015;21(4):309-319

5 Sundal C. Microglia: multiple roles in surveillance, circuit shaping, and response to injury. Neurology 2014;82(20):1846

6 Eyo UB, Dailey ME. Microglia: key elements in neural development, plasticity, and pathology. J Neuroimmune Pharmacol 2013;8(3):494-509

7 Loane DJ, Byrnes KR. Role of microglia in neurotrauma. Neurotherapeutics 2010;7(4):366-377 
8 Wolf SA, Boddeke HWGM, Kettenmann H. Microglia in physiology and disease. Annu Rev Physiol 2017;79(1):619-643

9 Streit WJ. Microglial-neuronal interactions. J Chem Neuroanat 1993;6(4):261-266

10 Chen Z, Trapp BD. Microglia and neuroprotection. J Neurochem 2016;136(Suppl 1) :10-17

11 Nayak D, Roth TL, McGavern DB. Microglia development and function. Annu Rev Immunol 2014;32(1):367-402

12 Michell-Robinson MA, Touil H, Healy LM, et al. Roles of microglia in brain development, tissue maintenance and repair. Brain 2015;138(Pt 5) :1138-1159

13 Streit WJ, Conde JR, Fendrick SE, Flanary BE, Mariani CL. Role of microglia in the central nervous system's immune response. Neurol Res 2005;27(7):685-691

14 Kierdorf K, Prinz M. Factors regulating microglia activation. Front Cell Neurosci 2013;7(April) :44

15 Lozano D, Gonzales-Portillo GS, Acosta S, et al. Neuroinflammatory responses to traumatic brain injury: etiology, clinical consequences, and therapeutic opportunities. Neuropsychiatr Dis Treat 2015;11:97-106

16 Das M, Mohapatra S, Mohapatra SS. New perspectives on central and peripheral immune responses to acute traumatic brain injury. J Neuroinflammation 2012;9(1):236

17 Mannix RC, Whalen MJ. Traumatic brain injury, microglia, and Beta amyloid. Int J Alzheimers Dis 2012;2012:608732

18 McGinn MJ, Povlishock JT. Pathophysiology of traumatic brain injury. Neurosurg Clin N Am 2016;27(4):397-407

19 Guerriero RM, Giza CC, Rotenberg A. Glutamate and GABA imbalance following traumatic brain injury. Curr Neurol Neurosci Rep 2015;15(5):27

20 Zhou X, He X, Ren Y. Function of microglia and macrophages in secondary damage after spinal cord injury. Neural Regen Res 2014;9(20):1787-1795

21 Chhor V, Moretti R, Le Charpentier T, et al. Role of microglia in a mouse model of paediatric traumatic brain injury. Brain Behav Immun 2017;63:197-209

22 Zhang Z, Zhang Z-Y, Wu Y, Schluesener HJ. Lesional accumulation of CD163+ macrophages/microglia in rat traumatic brain injury. Brain Res 2012;1461:102-110

23 Hernandez-Ontiveros DG, Tajiri N, Acosta S, Giunta B, Tan J, Borlongan CV. Microglia activation as a biomarker for traumatic brain injury. Front Neurol 2013;4:30
24 Donat CK, Scott G, Gentleman SM, Sastre M. Microglial activation in traumatic brain injury. Front Aging Neurosci 2017;9(JUN) :208

25 Rathbone ATL, Tharmaradinam S, Jiang S, Rathbone MP, Kumbhare DA. A review of the neuro- and systemic inflammatory responses in post concussion symptoms: Introduction of the "post-inflammatory brain syndrome" PIBS. Brain Behav Immun 2015;46:1-16

26 Balu R. Inflammation and immune system activation after traumatic brain injury. Curr Neurol Neurosci Rep 2014;14(10):484

27 Trahanas DM, Cuda CM, Perlman H, Schwulst SJ. Differential activation of infiltrating monocyte-derived cells after mild and severe traumatic brain injury. Shock 2015;43(3):255-260

28 Zhang G, Li Q, Wang L, et al. Traumatic brain injury induces macrophage subsets in the brain. Nephrol Dial Transplant 2013;34(1):202

29 Smith C. Review: the long-term consequences of microglial activation following acute traumatic brain injury. Neuropathol Appl Neurobiol 2013;39(1):35-44

30 Lull ME, Block ML. Microglial activation and chronic neurodegeneration. Neurotherapeutics 2010;7(4):354-365

31 Menzel L, Kleber L, Friedrich C, et al. Progranulin protects against exaggerated axonal injury and astrogliosis following traumatic brain injury. Glia 2017;65(2):278-292

32 Hanlon LA, Huh JW, Raghupathi R. Minocycline transiently reduces microglia/macrophage activation but exacerbates cognitive deficits following repetitive traumatic brain injury in the neonatal rat. J Neuropathol Exp Neurol 2016;75(3):214-226

33 Kovesdi E, Kamnaksh A, Wingo D, et al. Acute minocycline treatment mitigates the symptoms of mild blast-induced traumatic brain injury. Front Neurol 2012;3:111

34 Besson VC, Chen XR, Plotkine M, Marchand-Verrecchia C. Fenofibrate, a peroxisome proliferator-activated receptor alpha agonist, exerts neuroprotective effects in traumatic brain injury. Neurosci Lett 2005;388(1):7-12

35 Wang G, Jiang X, Pu H, et al. Scriptaid, a novel histone deacetylase inhibitor, protects against traumatic brain injury via modulation of PTEN and AKT pathway: scriptaid protects against TBI via AKT. Neurotherapeutics 2013;10(1):124-142 